\title{
Chapter 2 \\ Regulatory Mechanism of Neural \\ Progenitor Cells Revealed by Optical \\ Manipulation of Gene Expressions
}

\author{
Itaru Imayoshi, Mayumi Yamada, and Yusuke Suzuki
}

The basic-helix-loop-helix (bHLH) transcription factors Hes1, Ascl1/Mash1 and Olig2 facilitate the fate determination of astrocytes, neurons and oligodendrocytes, respectively (Imayoshi and Kageyama 2014). However, these bHLH transcription factors are co-expressed in multipotent self-renewing neural progenitor cells even before cell fate choice (Imayoshi et al. 2013). This finding indicates that these fate determination factors are differentially expressed between self-renewing and differentiating neural progenitor cells with unique expression dynamics. Live imaging analysis with fluorescent and bioluminescent proteins is a powerful strategy for monitoring expression dynamics. Our imaging results indicate that bHLH transcription factors are expressed in an oscillatory manner by neural progenitor cells, and that one of them becomes dominant in fate choice. We propose that the multipotent state of neural progenitor cells correlates with the oscillatory expression of several

Electronic Supplementary Material The online version of this chapter (https://doi. org/10.1007/978-981-13-7908-6_2) contains supplementary material, which is available to authorized users.

\footnotetext{
I. Imayoshi (四)

Graduate School of Biostudies, Kyoto University, Kyoto, Japan

Institute for Frontier Life and Medical Sciences, Kyoto University, Kyoto, Japan

World Premier International Research Initiative-Institute for Integrated Cell-Material Sciences, Kyoto University, Kyoto, Japan

The Hakubi Center, Kyoto University, Kyoto, Japan

Japan Science and Technology Agency, Precursory Research for Embryonic Science and Technology, Saitama, Japan

Medical Innovation Center/SK Project, Graduate School of Medicine, Kyoto University, Kyoto, Japan

e-mail: imayoshi.itaru.2n@kyoto-u.ac.jp; iimayosh@virus.kyoto-u.ac.jp
} 

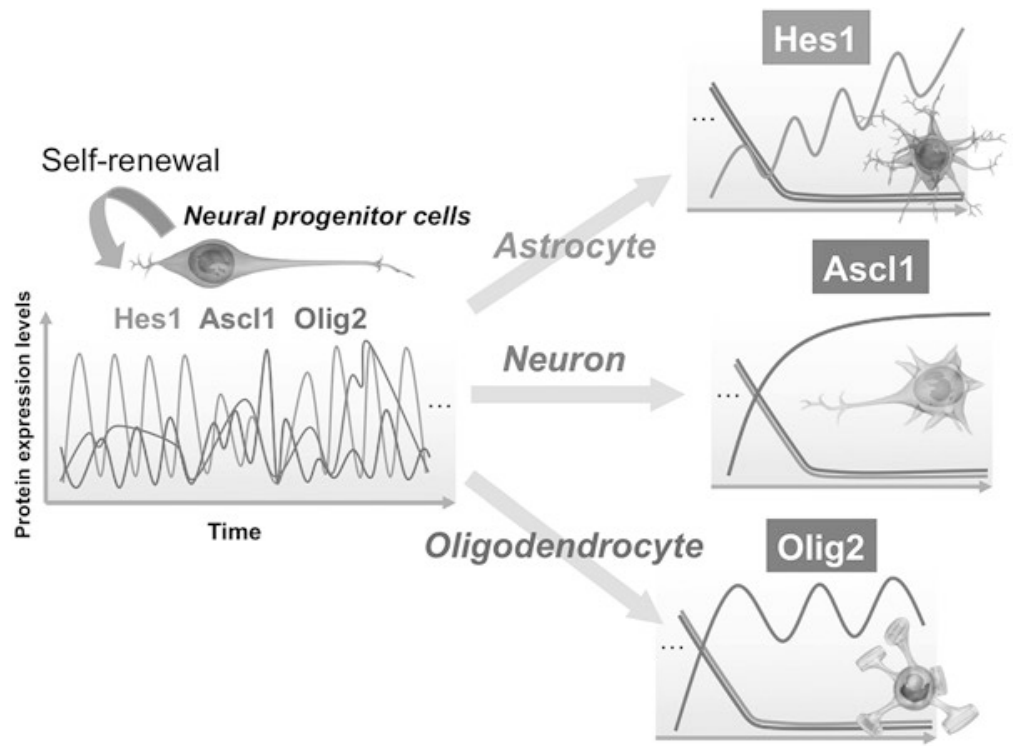

\section{Ascl1}

Fig. 2.1 Expression dynamics of bHLH factors in multipotency and cell fate determination. (This figure was modified from Figure 5 of Imayoshi and Kageyama 2014)

bHLH transcription factors, whereas the differentiated state correlates with the sustained expression of a single bHLH transcription factor.

To address the cousal relationships between the expression dynamics (oscillatory versus sustained) and functional outcomes (cell proliferation versus fate differentiation), the optogenetic approach has been employed to control the expression patterns of bHLH transcription factors (Imayoshi et al. 2013). We applied a novel optogenetic method (photo-activatable Gal4/UAS system) to manipulate the expression patterns of bHLH transcription factors using blue light illumination, showing that oscillatory expression activates the cell proliferation of neural progenitor cells, whereas sustained expression induces cell fate determination (Fig. 2.1).

M. Yamada

Graduate School of Biostudies, Kyoto University, Kyoto, Japan

Institute for Frontier Life and Medical Sciences, Kyoto University, Kyoto, Japan

World Premier International Research Initiative-Institute for Integrated Cell-Material

Sciences, Kyoto University, Kyoto, Japan

Medical Innovation Center/SK Project, Graduate School of Medicine, Kyoto University, Kyoto, Japan

Y. Suzuki

Graduate School of Biostudies, Kyoto University, Kyoto, Japan

Institute for Frontier Life and Medical Sciences, Kyoto University, Kyoto, Japan

Medical Innovation Center/SK Project, Graduate School of Medicine, Kyoto University, Kyoto, Japan 


\section{References}

Imayoshi I, Isomura A, Harima Y, Kawaguchi K, Kori H, Miyachi H, Fujiwara T, Ishidate F, Kageyama R (2013) Oscillatory control of factors determining multipotency and fate in mouse neural progenitors. Science 342:1203-1208

Imayoshi I, Kageyama R (2014) bHLH factors in self-renewal, multipotency, and fate choice of neural progenitor cells. Neuron 82:9-23

Open Access This chapter is licensed under the terms of the Creative Commons Attribution 4.0 International License (http://creativecommons.org/licenses/by/4.0/), which permits use, sharing, adaptation, distribution and reproduction in any medium or format, as long as you give appropriate credit to the original author(s) and the source, provide a link to the Creative Commons licence and indicate if changes were made.

The images or other third party material in this chapter are included in the chapter's Creative Commons licence, unless indicated otherwise in a credit line to the material. If material is not included in the chapter's Creative Commons licence and your intended use is not permitted by statutory regulation or exceeds the permitted use, you will need to obtain permission directly from the copyright holder.

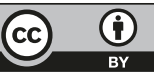

